\title{
Kavosh: an effective Map-Reduce-based association rule mining method
}

\author{
Mohammadhossein Barkhordari ${ }^{*}$ and Mahdi Niamanesh
}

\author{
*Correspondence: \\ Barkhordari@ictrc.ac.ir \\ Information \\ and Communication \\ Technology Research Center \\ No 5, Saeedi Alley, Colledge \\ Intersection, Enghelab Street, \\ Tehran 1599616313, Iran
}

\begin{abstract}
The immense amount of data generated on a daily basis by various devices and systems necessitates a change in data analysis methods. As an important part of analytics, data mining methods require a paradigm shift to solve problems because the old methods cannot manage massive data. Association rule mining is a data mining algorithm used to solve various domain problems. Because of the immense volume of data, one-node solutions are no longer useful, and it is necessary to solve problems by using a distributed and shared-nothing architecture such as Map-Reduce. However, when association rule mining is transferred to these architectures, new problems appear. The main problems are lack of data locality and iteration support and process skewness. In this paper, a method is proposed that solves these problems. Kavosh converts data into a unified format that helps nodes perform their tasks independently without the need to exchange data with other nodes. In addition, the proposed method compresses input data to facilitate data management. Another advantage is the lack of process skewness because it is possible to allocate a predefined amount of data to each node. Kavosh omits iterations required for finding frequent itemsets by changing the MapReduce architecture. The proposed method is implemented using Hadoop, and the results are compared with open-source products in terms of three aspects: execution time, load balancing and data compression. The results show that Kavosh outperforms other methods in these aspects.
\end{abstract}

Keywords: Big data, Data mining, Map-Reduce, Association rules

\section{Introduction}

With the growth of information, traditional analysis methods must be modified because they cannot handle immense amounts of data. Data mining algorithms are analytics that require a paradigm shift for algorithm execution and changes for deployment over nodes. Data mining algorithms must be modified so they can be executed over scalable and distributable environments. However, modifying data mining algorithms for distributed architecture is not easy. One problem with distributed architecture is data locality. This occurs when the required data for processing do not exist on the processor node.

One of the most prominent methods used to solve big data problems over sharednothing architecture is Map-Reduce. Map-Reduce [1] is used in open-source solutions such as Hadoop and Spark. There are many products in addition to Hadoop and Spark that can be used to solve data mining problems. However, pure Map-Reduce suffers from the data locality problem and does not support iteration. Because nearly all data

(c) The Author(s) 2018. This article is distributed under the terms of the Creative Commons Attribution 4.0 International License (http://creativecommons.org/licenses/by/4.0/), which permits unrestricted use, distribution, and reproduction in any medium, provided you give appropriate credit to the original author(s) and the source, provide a link to the Creative Commons license, and indicate if changes were made. 
mining algorithms are iterative in nature, it is necessary to solve the iteration problem in Map-Reduce to solve data mining problems by using Map-Reduce-based methods.

Association rule mining is a data mining algorithm that requires iteration to find frequent itemsets. Methods such as Apriori [2] and FP-Growth [3] solve association rule mining problems. There are two main parameters for association rule mining: confidence and support. A rule in an association rule is defined as $x \rightarrow y$ (if $x$, then $y$ ), where $\mathrm{x}$ and $\mathrm{y}$ are itemsets. The support of a rule is defined as the frequency of an itemset in a database. The confidence of a rule is defined as

$$
\text { Confidence }(x \rightarrow y)=\frac{\operatorname{Support}(\mathrm{x} \cap \mathrm{y})}{\operatorname{Support}(\mathrm{x})}
$$

In this paper, Kavosh, which is a method for association rule mining, is proposed. This method is designed for a shared-nothing architecture and can properly solve the association rule mining problem in Map-Reduce.

This method solves the data locality problem completely. Thus, the nodes do not require data from other nodes. By changing and unifying the data format, data compression and data load balancing are supported. In addition, iteration is omitted in the proposed method; therefore, it is well-suited for the Map-Reduce architecture.

The remainder of this paper is structured as follows: In "Related works" section, related works are discussed. In the third section, Kavosh is presented. The fourth section describes an evaluation, and the final section presents the conclusions.

\section{Related works}

Map-Reduce is used to solve many problems over a shared-nothing architecture. This method is also used to solve association rule mining. There are four main problems with association rule mining on a shared-nothing architecture, namely, lack of support for the following:

- Data locality,

- Iteration,

- Load balancing, and

- Data compression.

As mentioned above, the data locality problem is a lack of required data on the processor node, which creates data dependency among nodes. This problem causes network congestion and increases the algorithm execution time. Another problem is lack of iteration support. When intermediate results are created by the Reducers, they must be fed as input to the Mappers for another iteration. This problem also increases the execution time because intermediate data must be written on the disk by the Reducers and read by the Mappers to initiate another iteration. The third problem is the load balancing problem. This occurs when the processes are not allocated to the Mappers fairly. This problem reduces the algorithm speed because all nodes must wait for a busy node after job completion. The last problem is lack of support for data compression. Because of the large amount of data, data volume reduction is necessary for more rapid iterations and intermediate result storage. 
In some methods, traditional Apriori methods are used in Map-Reduce. Transactions are allocated to Mappers and frequent k-itemsets are extracted from each Mapper before the results are shuffled through combiners and the final k-itemsets are extracted according to support and confidence thresholds. In Oruganti et al. [4], Kovacs et al. [5], Li et al. [6], Mappers and Reducers are used, but in [7, 8], in addition to Mappers and Reducers, Combiners are used for better shuffling and to address performance issues.

In Lin et al [9], three methods are proposed: Single Pass Counting (SPC), Fixed Passes Combined-counting (FPC) and Dynamic Passes Combined-counting (DPC). In Apriori, each iteration must generally wait until the results of all the Reducers from the previous iteration have been generated.

The FiDoop [10] method uses three Map-Reduce phases to generate frequent itemsets. FiDoop claims to support automatic parallelization, load balancing, data distribution, and fault tolerance. ScaDiBino [11] extracts rules with the maximum length and one target field. This method omits iterations. In Yu et al. [12], the Distributed Parallel Apriori (DPA) algorithm is proposed and metadata are stored in the form of Transaction Identifiers (TIDs). In this method, a single scan of the database is required and a balanced workload among nodes is created.

Various proposed methods use FP-Growth on a shared-nothing architecture. In Li et al. [13], parallel FP-Growth is proposed for independently executing a group of tasks on a node. In Bechini et al. [14], MRAC and MRAC+ are proposed, which are MapReduce-based and FP-Growth-based methods, respectively, and FP-Growth was modified to overcome performance issues. In Yang et al. [15], a Hadoop-based method is proposed that uses a distributed DH-TRIE frequent pattern algorithm and tries to solve FP-Growth problems for big data. In Tlili et al. [16], a partitioning method is used to achieve load balancing problems for association rule mining. PARMA [17] creates multiple small random samples of the input data and runs a mining algorithm on them. Because it is implemented on small samples, the algorithm can be run in parallel and independently. The results are aggregated to produce the final results. In Yu and Zhou [18], Tidset-based Parallel FP-tree (TPFP-tree) and Balanced Tidset-based Parallel FPtree (BTP-tree) are proposed. In the proposed method, a transaction identification set (Tidset) is used to provide direct access to transactions instead of a full database scan. In Moens et al. [19], two methods are proposed: Dist-Eclat and BigFIM. Dist-Eclat has three steps: finding the frequent items, generating frequent itemsets of size $k$ and subtree mining. BigFIM also has three steps: generating frequent itemsets of size $\mathrm{k}$, finding potential extensions and sub-tree mining. The Sequence-Growth algorithm is designed according to the concepts of the lexicographical sequence tree and the lazy mining pruning strategy and is implemented in the MapReduce framework for a distributed execution [20]. In Liang et al. [21], an algorithm for lexicographic frequent itemset generation is proposed, which claims to find the maximum information from a database regarding frequent itemsets and their respective frequencies in a single database scan.

\section{Methods}

The proposed method uses the Map-Reduce architectural structure for association rule mining. The first step in the proposed method is to convert the input data items to a Kavosh format and the second step is to extract rules with variable lengths. In this paper, 
the number of fields after the "if" conditions are applied is defined as the length of the rule. In the proposed method, data are converted into Kavosh format, which helps nodes execute their processes independently. This format is a unified format and other input data formats are converted to this format. The proposed unified format helps create $<$ key, value $>$ pairs for use in the Map-Reduce method.

\section{- Converting input data items to Kavosh format}

In this section, the Kavosh format is defined

$\Theta=\left\{\theta_{1}, \theta_{2}, \ldots, \theta_{\mathrm{n}}, f\right\}$.

The input data table is denoted as $\Theta$, the columns of the table as $\theta$ and the table key as $f$

$\theta_{\mathrm{k}}=\left\{\mu_{1}, \mu_{2}, \ldots, \mu_{\mathrm{m}}\right\}$

The distinct values of each column are denoted as $\mu$. In this paper, it is assumed that all $\theta$ values are discrete and that a continuous $\theta$ must be converted to discrete values.

Based on the above definitions, the Kavosh format is defined as follows:

$$
\mathrm{F}=\left\{\theta_{1} \rightarrow \mu_{1}, \theta_{2} \rightarrow \mu_{2}, \ldots, \theta_{\mathrm{n}} \rightarrow \mu_{\mathrm{m}}\right\}
$$

where $\mathrm{F}$ is the Kavosh table and each $\theta_{\mathrm{p}} \rightarrow \mu_{\mathrm{q}}$ is equal to zero or one. Each input column value is converted to a Boolean column if there are more than two values for the column.

The input data $(\Theta)$ are divided into equal segments. For fragmentation, the function $\psi$ is used, which is defined as follows:

$$
\Psi\left(F, f_{\text {start }}, f_{\text {finish }}, \eta_{k}\right)
$$

where $\eta_{k}$ is the Mapper node to which the data $F$ are allocated from key $f_{\text {start }}$ to key $f_{\text {finish }}$. From the previous conversion steps, a Boolean matrix is created on each Mapper. Each field $\mathrm{F}$ can be selected as a target field. If a binary position is assigned to each field $\mathrm{F}$ (except target fields), each row can be converted into a decimal number. Therefore, a $<$ Key, Value $>$ pair is created in which the Key is the decimal number of each row (RuleKey) and the Value is the decimal value of the target field. In the next step, the Kavosh triple is created, which is defined as follows:

\section{$<$ Rule-Key, Rule count, Target field count with the specified value $>$}

According to the generated <Rule-Key, Target fields $>$, the count of each Rule-Key and target field with the specified value is calculated and the Kavosh triple set is created. To convert Mapper data into the Kavosh triple and Rule-Key aggregation, the function $T$ is used.

$$
T\left(\eta_{k}, \rho_{k}\right)
$$

where $\rho_{k}$ is the result of computation of the input data. After calculating $\rho_{k}$ for each Mapper, all $\rho_{k}$ are reduced to $\rho_{\text {Reducer }}$ by the function $£$.

$$
£\left(\rho_{1}, \rho_{2}, \ldots, \rho_{\mathrm{p}}, \rho_{\text {Reducer }}, 9\right)
$$

where 9 is the Reducer node and $\mathrm{p}$ is the number of Mappers. 


\section{- Rule extraction}

In this section, a Map-Key is added to the Rule-Key (м). The Map-Key is used for rule generation, and its length in binary format is equal to the Rule-Key. Here, $\rho_{\text {Reducer }}$ is sent to the rule generation nodes. Each rule generation node has one or more Map-Keys. The function $\varpi$ allocates $\rho_{\text {Reducer }}$ to each rule generation node.

where $\mathrm{t}$ is the Map-Key, $\mathrm{q}$ is the number of Map-Keys allocated to a rule generation node, and $\mathrm{b}$ is the rule generation node.

$\varpi\left(\varrho_{\text {Reducer }}, \mathrm{l}_{1}, \mathrm{l}_{2}, \ldots, \mathrm{l}_{\mathrm{q}}, \mathrm{b}_{r}\right)$

Each Map-Key is concatenated to all Rule-Keys. If the length of the Rule-Key in binary format is equal to $\mathrm{L}$, then there are $2^{\mathrm{L}}-1$ Map-Keys. They start from zero to $2^{\mathrm{L}}-1$ and are concatenated with the Rule-Keys to create various condition combinations of fields $\mathrm{F}$. The concatenation $(Y)$ of the Map-Key with the result of the "logical and" $(\varkappa)$ of the MapKey and the Rule-Key is called MHB_Key, and $\tau$ is the MHB_Key.

$\tau=r\left(\mathrm{t}_{\mathrm{i}}, \mathcal{N}\left(\mathrm{M}, \mathrm{t}_{\mathrm{i}}\right)\right)$

There are two important factors for rule extraction in association rule mining: confidence and support. In this paper, Д $(\tau)$ is defined as the frequency of a specified $M H B_{-}$ Key $(\tau)$ and $\zeta(\tau, \sigma, \rho)$ is defined as the frequency of a specific decimal value $(\rho)$ that is equal to the target field $(\sigma)$ for a specified $M H B_{-} K e y(\tau)$. In addition, $\varsigma$ is the total number of generated Kavosh triple sets. Thus, the support (२) is calculated using the following formula:

$$
\tau(\tau)=\frac{Д(\tau)}{\varsigma}
$$

Moreover, the confidence (C) is calculated using the following formula:

$$
\mathrm{C}(\tau, \sigma, \rho)=\frac{\zeta(\tau, \sigma, \rho)}{\text { Д }(\tau)}
$$

After2 andc have been calculated, they are compared with the defined thresholds for the association rules and the final results are produced. The rules are extracted using Д.

$$
\text { Д (ट( } \left.\tau), \mathrm{C}(\tau, \sigma), \alpha, \beta, \bigsqcup_{r}\right)
$$

where $\alpha$ is the support threshold and $\beta$ is the confidence threshold. Each rule generation node creates its final result separately because the results do not have common rules with other nodes. Figure 1 shows the Kavosh architecture. It consists of three layers: the Mapper layer, the Reducer layer and the rule generation layer.

For clearer illustration, pseudocodes are provided. Table 1 lists the functions used in the code. 


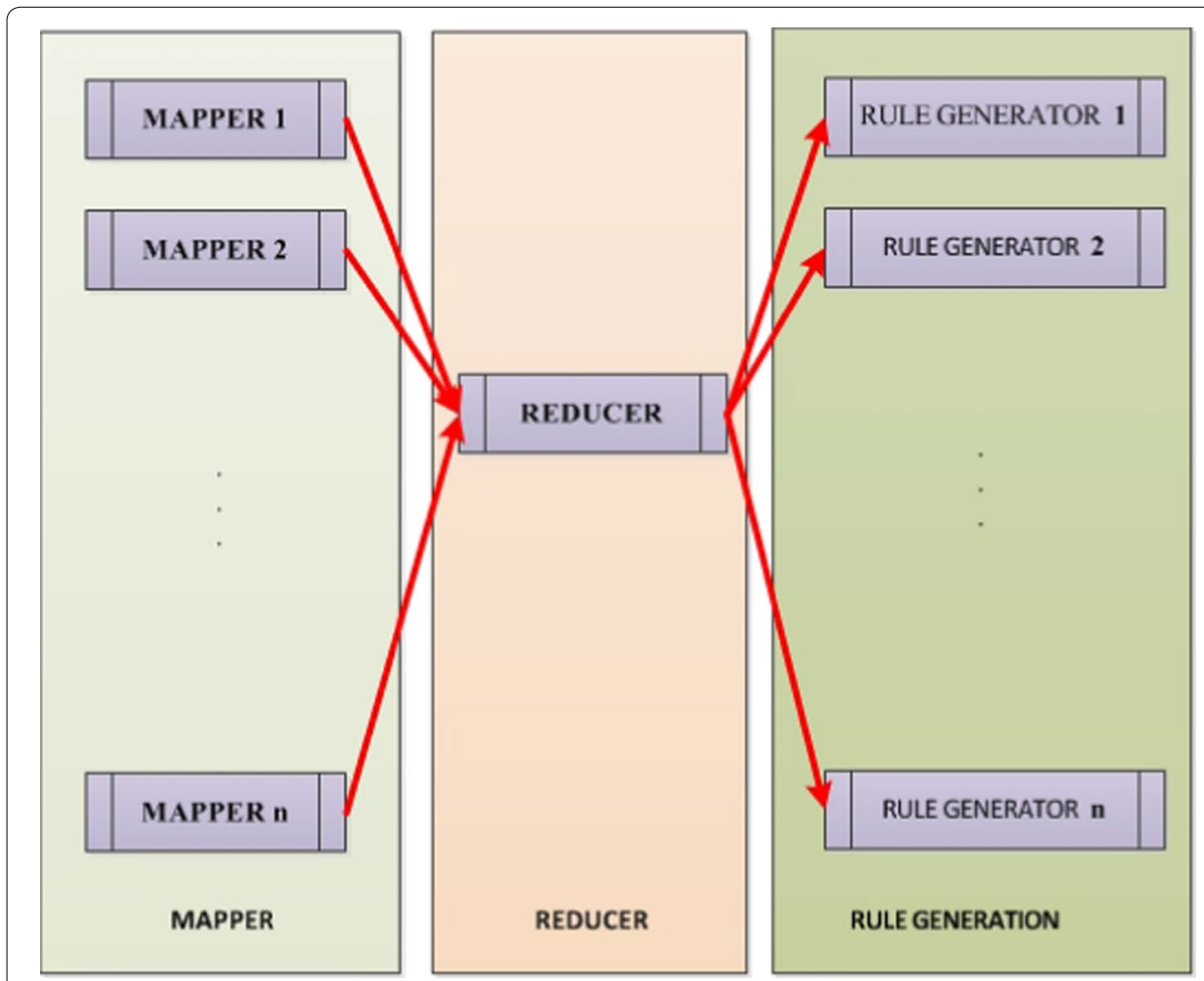

Fig. 1 Kavosh architecture

Table 1 Pseudocode functions

\begin{tabular}{|c|c|}
\hline Function & Description \\
\hline NOC(TableName) & Returns the number of columns \\
\hline NOR(TableName) & Returns the number of rows \\
\hline NODV(TableName, ColumnName/ColumnID) & Returns the number of distinct values in the specified column \\
\hline NaOC(TableName, k) & Returns the name of the kth column in the specified table \\
\hline DVC(TableName,ColumnName/ColumnID, k) & Returns the kth distinct value of the specified column \\
\hline CT(ColumnsArray [,TableName) & $\begin{array}{l}\text { Creates a table with an input column array with a specified table } \\
\text { name }\end{array}$ \\
\hline RVT(TableName, ColumnName/ColumnID,RowID) & $\begin{array}{l}\text { Returns the value of the specified table name, column name/ } \\
\text { column ID, and row ID }\end{array}$ \\
\hline EXEC (Query, ResultTable) & $\begin{array}{l}\text { Executes a query, creates a results table and puts the results into } \\
\text { the results table } \\
\text { The results table can be created in RAM or HDD, according to the } \\
\text { node hardware specification. }\end{array}$ \\
\hline \& & \& is a logical operator (And) \\
\hline 12B(i) & Converts the integer value i to a binary value \\
\hline
\end{tabular}


The following code shows the binominal conversion.

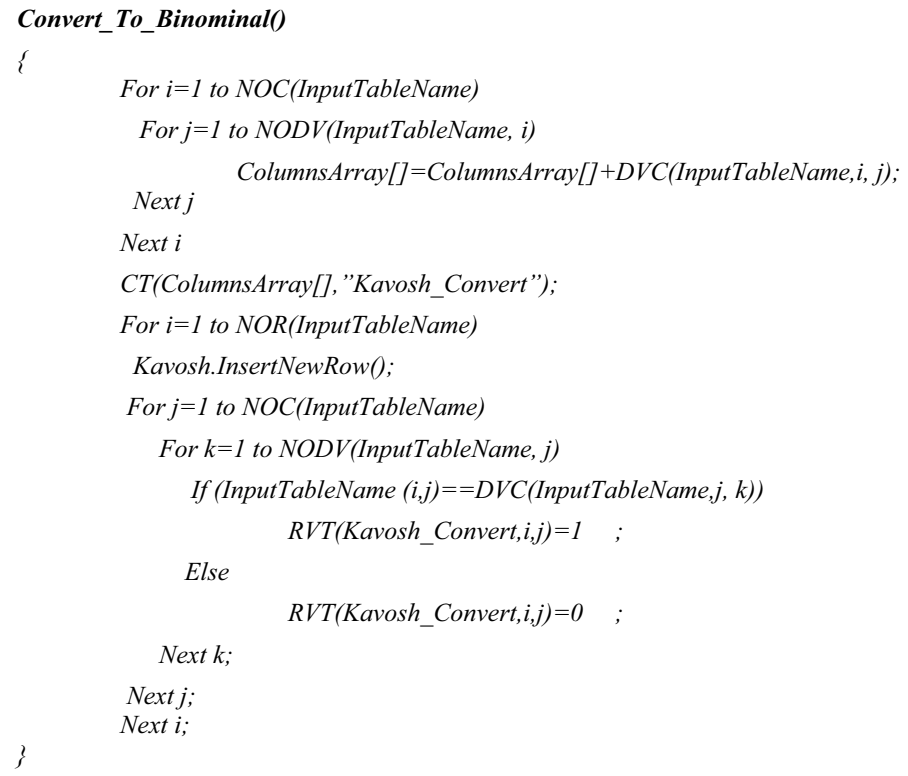

The following pseudocode shows the Mapper's functionality:

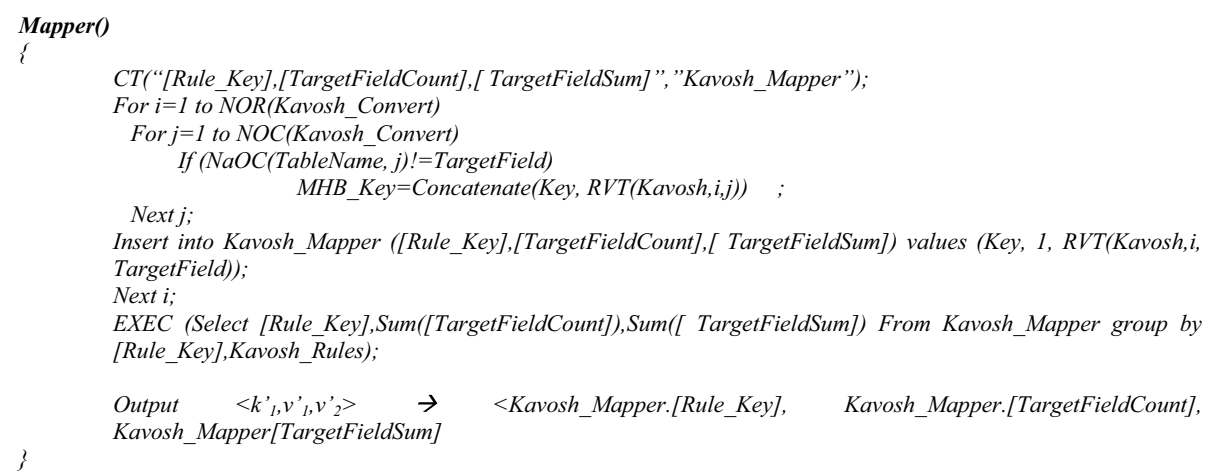

In the reduce phase, the $\operatorname{EXEC}()$ function is repeated to obtain the final intermediate results layer. The following pseudocode shows the Reducer phase:

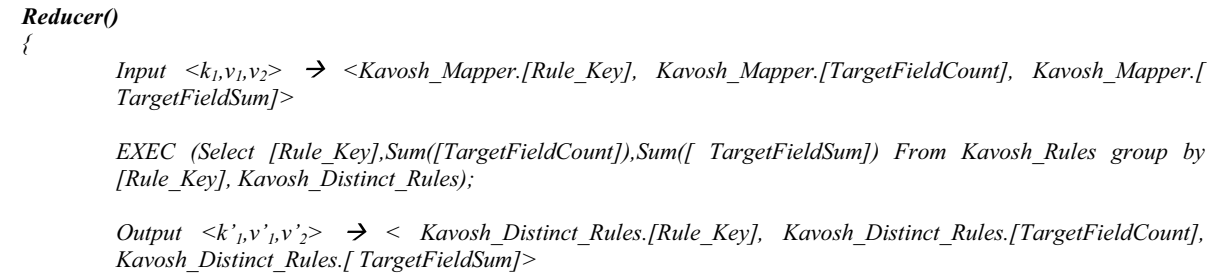


Table 2 Sample input information

\begin{tabular}{lllll}
\hline City & Sex & Income & New service & Mapper \\
\hline Tehran & Male & High & Yes & 1 \\
Tehran & Female & Low & Yes & \\
Yazd & Male & High & No & 2 \\
Yazd & Male & High & Yes & \\
Tehran & Female & Low & No & \\
Yazd & Male & High & No & \\
\hline
\end{tabular}

Table 3 Binominal format

\begin{tabular}{lllll}
\hline Tehran & Sex & High & New service & Mapper \\
\hline 1 & 1 & 1 & 1 & 1 \\
1 & 0 & 0 & 1 & \\
0 & 1 & 1 & 0 & 2 \\
0 & 1 & 1 & 1 & \\
1 & 0 & 0 & 0 & \\
0 & 1 & 1 & 0 & \\
\hline
\end{tabular}

After the extraction of all the rules with a maximum length in the intermediate results layer (Fig. 4), a mapping field is added to these rules. Each rule is converted to $2^{\mathrm{n}}-1$ rules, where $\mathrm{n}$ is the number of input data item fields. The new rule value is equal to the concatenation of the mapping field and the result of the logical And between the mapping field and the rule value.

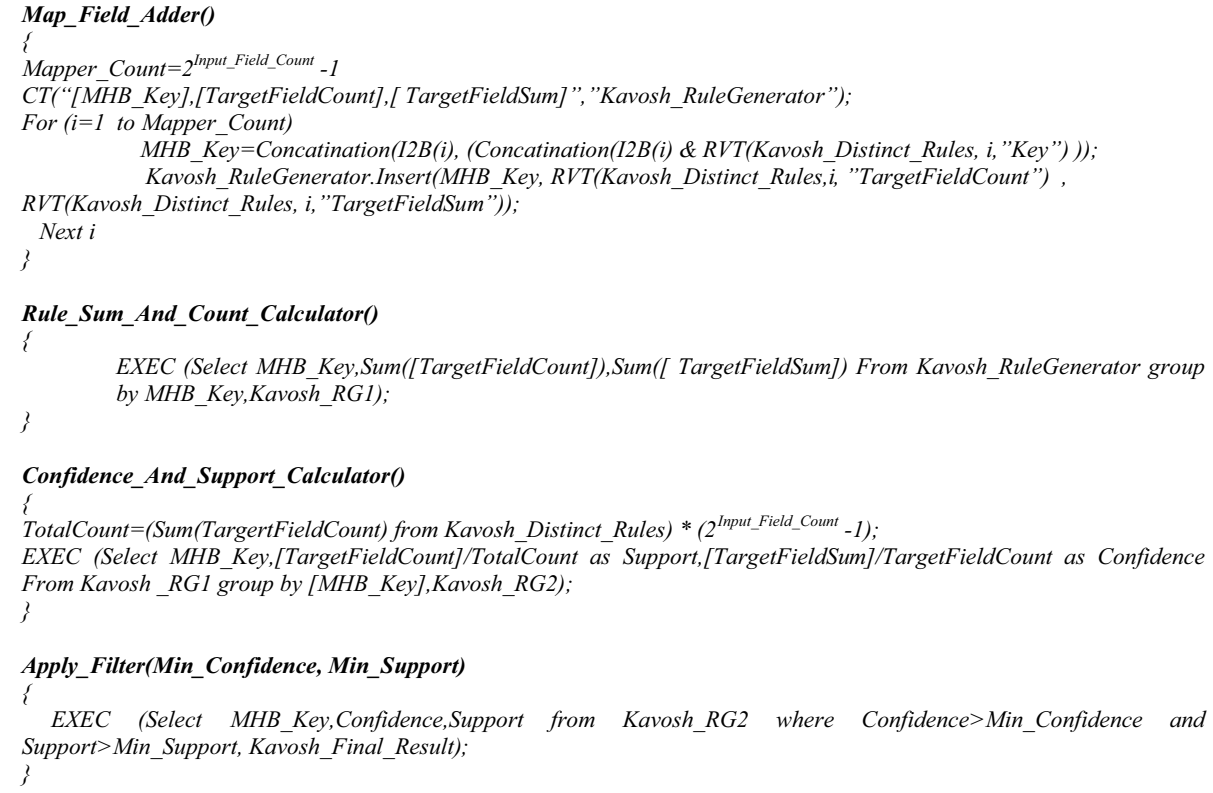

Suppose the input data are as described in Table 2, where New Service is the target field and the data are divided between two Mappers. 
Table 4 Mapper results

\begin{tabular}{lllll}
\hline Decimal rule & Target field & Rule count & Rule sum & Mapper \\
\hline 7 & 1 & 1 & 1 & 1 \\
4 & 1 & 1 & 1 & \\
3 & 0 & 1 & 0 & 2 \\
3 & 0 & 1 & 0 & \\
4 & 1 & 1 & 1 & \\
3 & 0 & 1 & 0 & \\
\hline
\end{tabular}

Table 5 Reducer results

\begin{tabular}{lll}
\hline Rule & Rule count & Rule sum \\
\hline 7 & 1 & 1 \\
4 & 2 & 2 \\
3 & 3 & 0 \\
\hline
\end{tabular}

Table 6 Mapping field addition stage

\begin{tabular}{|c|c|c|c|c|c|}
\hline Mapping field & Rule & MHB_Key (Binary) & $\begin{array}{l}\text { MHB_Key } \\
\text { (Decimal) }\end{array}$ & Rule count & Rule sum \\
\hline 111 & 111 & 111111 & 63 & 1 & 1 \\
\hline 111 & 100 & 111100 & 60 & 2 & 2 \\
\hline 111 & 011 & 111011 & 59 & 3 & 0 \\
\hline 110 & 111 & 110110 & 54 & 1 & 1 \\
\hline 110 & 100 & 110100 & 52 & 2 & 2 \\
\hline 110 & 011 & 110010 & 50 & 3 & 0 \\
\hline 101 & 111 & 101101 & 45 & 1 & 1 \\
\hline 101 & 100 & 101100 & 44 & 2 & 2 \\
\hline 101 & 011 & 101001 & 41 & 3 & 0 \\
\hline 100 & 111 & 100100 & 36 & 1 & 1 \\
\hline 100 & 100 & 100100 & 36 & 2 & 2 \\
\hline 100 & 011 & 100000 & 32 & 3 & 0 \\
\hline 011 & 111 & 011011 & 27 & 1 & 1 \\
\hline 011 & 100 & 011000 & 24 & 2 & 2 \\
\hline 011 & 011 & 011011 & 27 & 3 & 0 \\
\hline 010 & 111 & 010010 & 18 & 1 & 1 \\
\hline 010 & 100 & 010000 & 16 & 2 & 2 \\
\hline 010 & 011 & 010010 & 18 & 3 & 0 \\
\hline 001 & 111 & 001001 & 9 & 1 & 1 \\
\hline 001 & 100 & 001000 & 8 & 2 & 2 \\
\hline 001 & 011 & 001001 & 9 & 3 & 0 \\
\hline
\end{tabular}

Table 3 shows the data after conversion to a binominal format. Table 4 shows the Mapper results. The rules are now combined based on their keys in the Reducer layer. As shown in Table 5, the rules are combined in this phase, and their rule count and rule summation values are recalculated. Then, the mapping fields are added to the 
Table 7 Rule sum and count recalculation

\begin{tabular}{llll}
\hline MHB_Key (Binary) & MHB_Key (Decimal) & Rule count & Rule sum \\
\hline 111111 & 63 & 1 & 1 \\
111100 & 60 & 2 & 2 \\
111011 & 59 & 3 & 0 \\
110110 & 54 & 1 & 1 \\
110100 & 52 & 2 & 2 \\
110010 & 50 & 3 & 0 \\
101101 & 45 & 1 & 1 \\
101100 & 44 & 2 & 2 \\
101001 & 41 & 3 & 0 \\
100100 & 36 & 3 & 3 \\
100000 & 32 & 3 & 0 \\
011011 & 27 & 4 & 1 \\
011000 & 24 & 2 & 2 \\
010010 & 18 & 4 & 1 \\
010000 & 16 & 2 & 2 \\
001001 & 9 & 4 & 2 \\
001000 & 8 & 2 & \\
\hline
\end{tabular}

Table 8 Support and confidence results

\begin{tabular}{lcc}
\hline Rule & Support (\%) & $\begin{array}{c}\text { Confidence } \\
\text { (one) (\%) }\end{array}$ \\
\hline 63 & 2 & 100 \\
60 & 5 & 100 \\
59 & 7 & 0 \\
54 & 2 & 100 \\
52 & 5 & 100 \\
50 & 7 & 0 \\
45 & 2 & 100 \\
44 & 5 & 100 \\
41 & 7 & 0 \\
36 & 7 & 100 \\
32 & 7 & 0 \\
27 & 10 & 20 \\
24 & 5 & 100 \\
18 & 10 & 20 \\
16 & 5 & 100 \\
9 & 10 & 20 \\
8 & 5 & 100 \\
\hline
\end{tabular}

rules. The mapping fields are sorted to prevent future shuffling among the Mappers. As the input item, the field count is equal to three, and seven mapping fields $\left(2^{3}-1\right)$ must be added to the rules. Table 6 shows this stage.

The rule sum and count are recalculated, and the same key rules are combined, as depicted in Table 7. In the next stage, confidence and support are calculated. Table 8 shows the results of this stage. As mentioned earlier, confidence values must be calculated for two values: zero and one. Finally, the Reduce stage of the rule generation 
Table 9 Reducer results

\begin{tabular}{llc}
\hline Rule & Support (\%) & $\begin{array}{l}\text { Confidence } \\
\text { (one) (\%) }\end{array}$ \\
\hline 59 & 7 & 0 \\
50 & 7 & 0 \\
41 & 7 & 0 \\
36 & 7 & 100 \\
32 & 7 & 0 \\
\hline
\end{tabular}

layer filters rules according to thresholds for support and confidence. If support is greater than $5 \%$ or confidence is greater than $95 \%$, the Reducer results are as shown in Table 9. As shown in Table 9, some rows have confidence equal to 0\%. These rows have confidence equal to $100 \%$ for a zero value. In other words, for rule filtering with confidence greater than $95 \%$, it is necessary to consider confidence lower than $5 \%$ $(100-95 \%)$. Kavosh can be summarized as follows:

- The input data are converted to a binominal format.

- The converted data are distributed among the Mappers.

- The results of the Mappers are sent to the Reducer layer.

- Mapping fields are added to the results of the Reducer layer, and MHB_Keys are generated.

- The Reducer results are sent to the Rule generation layer.

- Confidence and support parameters are applied to the extracted rules to create the final results.

\section{Evaluation}

The proposed method is evaluated on the TPC-DS dataset and a real-world dataset. "The TPC Benchmark DS (TPC-DS) is a decision support benchmark that models several generally applicable aspects of a decision support system, including queries and data maintenance. The benchmark provides a representative evaluation of performance as a general purpose decision support system. A benchmark result measures query response time in single user mode, query throughput in multi user mode and data maintenance performance for a given hardware, operating system, and data processing system configuration under a controlled, complex, multi-user decision support workload. The purpose of TPC benchmarks is to provide relevant, objective performance data to industry users. TPC-DS Version 2 enables emerging technologies, such as Big Data systems, to execute the benchmark" (http://www.tpc.org/tpcds).

The proposed method can use DBMS on each node independently. PostgreSQL is used as DBMS on each node. PostgreSQL is a powerful, open-source object-relational database system that uses and extends the SQL language, combined with many features that safely store and scale the most complicated data workloads (https://www. postgresql.org/about/). 
To achieve higher performance, an In-Memory database is used. Redis is an opensource (BSD licensed), in-memory data structure store, which is used as a database, cache and message broker (https://redis.io/).

\section{TPC-DS}

To implement the Kavosh method, Hadoop 2.7.3 is used. Ubuntu 16.04 is installed on each node. Parts of Hadoop were modified for Kavosh implementation. As described above, there are three layers of nodes in Kavosh: The first layer consists of the Mapper nodes, the second layer consists of the Reducer node, and the third layer is the rule generation layer. The DataNodes of Hadoop are used for these three layers. A MetaNode is added to the Hadoop NameNode to maintain the type information of each group of nodes. Because of the Kavosh data format and the independence of each node, it is possible to use a database management system for each data node. Therefore, PostgreSQL 9.6.1 is used for the Mapper nodes. A Kavosh converter exists on the Mapper nodes to convert the input data format into the Kavosh format. To achieve faster computation speed, an in-memory database is used for the Reducer node and the rule generation nodes. Redis 3.2.5 is used as an in-memory database on these nodes with AOF disk persistence for the Reducer and disk persistence is disabled for the rule generation nodes. Table 10 shows DBMS for the various node types.

Figure 2 shows Kavosh architecture using Hadoop.

For data generation, TPC-DS_Tools_v2.8.0 was used. Four tables (Customer_demographics, Store_sales, Web_sales, and Item) were used for association rule mining. Figures 3 and 4 show the ER-Diagrams.

A database view is created over four mentioned tables. This view contains customer information and items that each customer bought. Figures 5, 6, 7 and 8 show the table structures.

The Kavosh method extracts market basket analysis (MBA) rules for items on various sell channels. The frequent items with a maximum length of twenty are extracted. In other words, twenty related items are extracted. The scale factor is $100 \mathrm{~TB}$ and $\mathrm{SF}=100,000$. Table 11 shows the number of rows in each data table.

Tables 12 and 13 show the node specifications for the Kavosh method. A total of 86 nodes are used for evaluation. Seventy nodes were used as the Mapper nodes, one node was used as the Reducer node and fifteen nodes were used as the rule generation nodes. The input data must be converted to the Kavosh format and it requires $6740 \mathrm{~s}$ for the Kavosh converter to convert the input data to the Kavosh format. Each Mapper node simultaneously converts approximately 1.5 TB of data.

Table 10 DBMS for the various node types

\begin{tabular}{ll}
\hline Node type & DBMS \\
\hline Mapper & PostgreSQL \\
Reducer & Redis \\
Rule generation & Redis \\
\hline
\end{tabular}



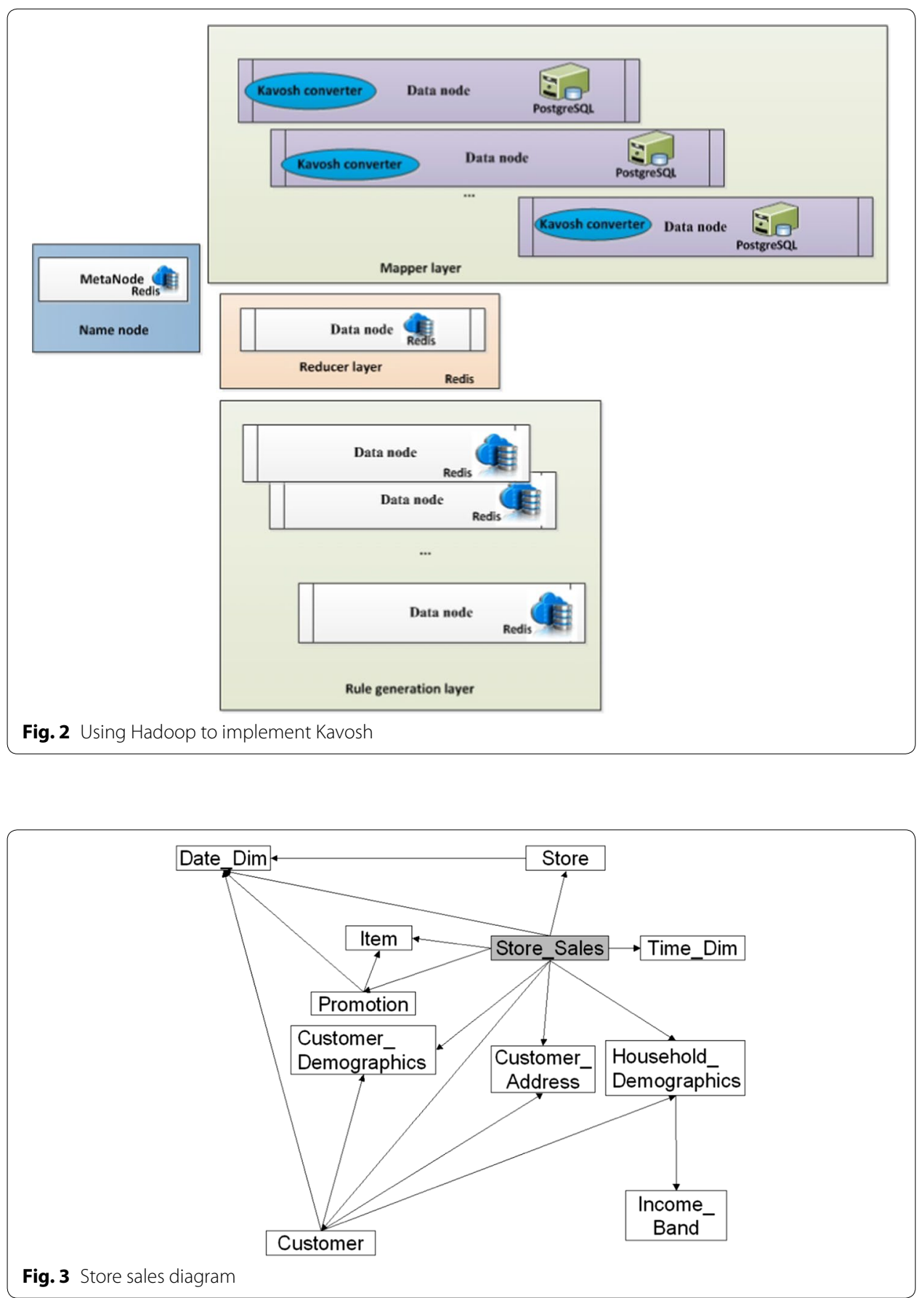

The Kavosh method is compared with FiDoop [10] and Sequence-Growth [20]. Table 14 shows the node specifications for the FiDoop and Sequence-Growth methods.

The total CPU, RAM and HDD used for Kavosh and other methods are equal.

To evaluate the three methods, frequent items for MBA of web sales and store sales are extracted. The association rule parameters are shown in Table 15. 


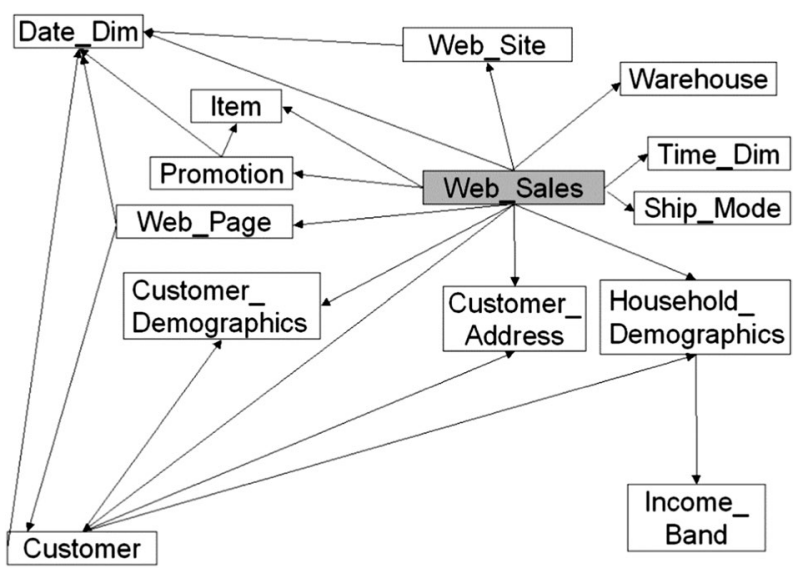

Fig. 4 Web sales diagram

\begin{tabular}{|l|l|l|l|l|}
\hline Column & Datatype & NULLs & Primary Key & Foreign Key \\
\hline ss_sold_date_sk & identifier & & & d_date_sk \\
\hline ss_sold_time_sk & identifier & & & t_time_sk \\
\hline ss_item_sk(1) & identifier & N & Y & i_item_sk \\
\hline ss_customer_sk & identifier & & & c_customer_sk \\
\hline ss_cdemo_sk & identifier & & & cd_demo_sk \\
\hline ss_hdemo_sk & identifier & & & hd_demo_sk \\
\hline ss_addrsk & identifier & & & ca address_sk \\
\hline ss_store_sk & identifier & & & s_store_sk \\
\hline ss_promo_sk & identifier & & & P_promo_sk \\
Fig.5 Store_sales & & & \\
\hline
\end{tabular}

\begin{tabular}{|l|l|l|l|l|}
\hline Column & Datatype & NULLs & Primary Key & Foreign Key \\
\hline ws_sold_date_sk & identifier & & & d_date_sk \\
\hline ws_sold_time_sk & identifier & & & t_time_sk \\
\hline ws_ship_date_sk & identifier & & & d_date_sk \\
\hline ws_item_sk(1) & identifier & N & Y & i_item_sk \\
\hline ws_bill_customer_sk & identifier & & & c_customer_sk \\
\hline ws_bill_cdemo_sk & identifier & & & cd_demo_sk \\
\hline ws_bill_hdemo_sk & identifier & & & hd_demo_sk \\
\hline ws_bill_addr_sk & identifier & & & ca_address_sk \\
\hline ws_ship_customer_sk & identifier & & c_customer_sk \\
\hline ws_ship_cdemosk & identifier & & & cd_demo_sk \\
\hline ws_ship_hdemo_sk & identifier & & & hd_demo_sk \\
\hline ws_ship_addr_sk & identifier & & ca_address_sk \\
\hline ws_web_page_sk & identifier & & & wp_web_page_sk \\
\hline ws_web_site_sk & identifier & & & web_site_sk \\
\hline ws_ship_mode_sk & identifier & & sm_ship_mode_sk \\
\hline ws_warehousessk & identifier & & w_warehouse_sk \\
\hline ws_promo_sk & identifier & & & p_promo_sk \\
Fig. 6 web_sales & & & \\
\hline
\end{tabular}

They are compared with one another in three dimensions: execution time, data compression and load balancing.

\section{Execution time}

The method proposed by FiDoop requires three Map-Reduce cycles to generate the final results. The execution time for each cycle is almost the same, and in each cycle, a full database scan is required. Sequence-Growth requires twenty Map-Reduce cycles 


\begin{tabular}{|l|l|l|l|l|}
\hline \multicolumn{9}{|c|}{} & Datatype & NULLs & Primary Key & Foreign Key \\
\hline Column & identifier & N & Y & \\
\hline i_item_sk & char(16) & N & & \\
\hline i_item_id (B) & date & & & \\
\hline i rec start date & date & & & \\
\hline i_rec_end_date & varchar(200) & & & \\
\hline i_item_desc & decimal(7.2) & & & \\
\hline i_current_price & decimal(7.2) & & & \\
\hline i_wholesale_cost & integer & & & \\
\hline i_brand_id & char(50) & & & \\
\hline i_brand & integer & & & \\
\hline i_class_id & char(50) & & & \\
\hline i_class & integer & & & \\
\hline i_category_id & char(50) & & & \\
\hline i_category & integer & char(50) & & \\
\hline i_manufact_id & char(20) & & & \\
\hline i_manufact & char(20) & & & \\
\hline i_size & char(20) & & & \\
\hline i_formulation & char(10) & & & \\
\hline i_color & char(10) & & & \\
\hline i_units & integer & & & \\
\hline i_container & char(50) & & \\
\hline i_manager_id & & & \\
\hline i__product_name & & & \\
Fig.7 Items & & & & \\
\hline
\end{tabular}

\begin{tabular}{|c|c|c|c|c|}
\hline Column & Datatype & | NULLs & Primary Key & | Foreign Key \\
\hline cd_demo_sk & identifier & $\mathrm{N}$ & $\mathrm{Y}$ & \\
\hline cd_gender & char(1) & & & \\
\hline cd_marital_status & char(1) & & & \\
\hline cd_education_status & char $(20)$ & & & \\
\hline cd purchase estimate & & & & \\
\hline cd_credit_rating & $\frac{1}{\text { charet }(10)}$ & & & \\
\hline cd dep count & integer & & & \\
\hline cd_dep_employed_count & $\begin{array}{l}\text { inceger } \\
\text { integer } \\
\end{array}$ & & & \\
\hline cd_dep_college_count & integer & & & \\
\hline
\end{tabular}

Fig. 8 Customer_Demographics

Table 11 Number of rows in each data table

Table name

Items

web_sales

Store_sales

Customer_Demographics
Number of rows

Table 12 Mapper node specifications

\begin{tabular}{ll}
\hline CPU & $\begin{array}{l}\text { Intel Core i7-3770 } \\
\text { Quad-Core Processor } \\
\end{array}$ \\
\hline 3.4 GHz \\
\hline RAM & $2 \mathrm{~TB}$ \\
\hline
\end{tabular}

Table 13 Reducer and rule generation node specifications

\begin{tabular}{ll}
\hline CPU & $\begin{array}{l}\text { Intel Core i7-3770 } \\
\text { Quad-Core Processor } \\
\mathbf{3 . 4 G H z}\end{array}$ \\
\hline HDD & $500 \mathrm{~GB}$ \\
RAM & $512 \mathrm{~GB}$ \\
\hline
\end{tabular}


Table 14 Nodes for FiDoop and Sequence-Growth

\begin{tabular}{ll}
\hline CPU & $\begin{array}{l}\text { Intel Core i7-3770 } \\
\text { Quad-Core Processor } \\
\mathbf{3 . 4} \mathbf{G H z}\end{array}$ \\
\hline HDD & $1.5 \mathrm{~TB}$ \\
RAM & $150 \mathrm{~GB}$ \\
\hline
\end{tabular}

Table 15 Association rule parameters

\begin{tabular}{ll}
\hline Parameter name & $\begin{array}{l}\text { Parameter } \\
\text { value (\%) }\end{array}$ \\
\hline Confidence & 90 \\
Support & 0.05 \\
\hline
\end{tabular}

Table 16 Execution details

\begin{tabular}{lccc}
\hline Method name & Map (s) & Reduce (s) & $\begin{array}{l}\text { Rule } \\
\text { generation } \\
\text { (s) }\end{array}$ \\
\hline FiDoop & 1265 & 1194 & 0 \\
Sequence-Growth & 3500 & 2890 & 0 \\
Kavosh & 288 & 265 & 1159 \\
\hline
\end{tabular}

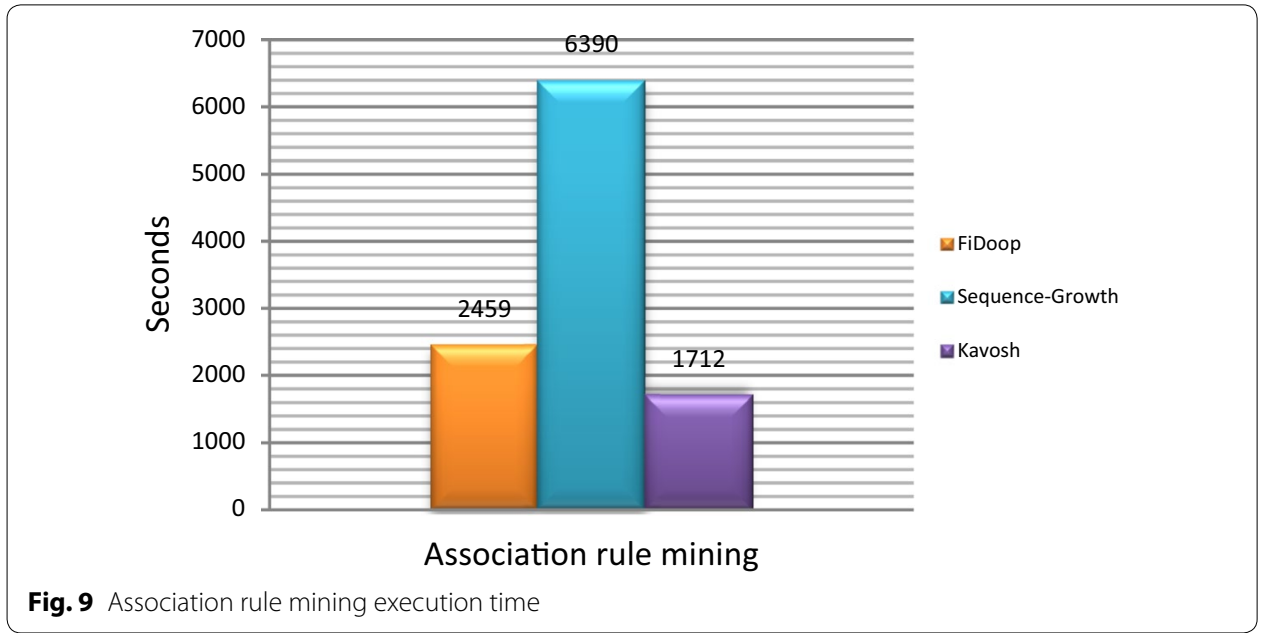

because it generates each frequent item in a Map-Reduce cycle. However, the execution time decreases each cycle in comparison with the previous cycle because the number of frequent items decreases. Nevertheless, Kavosh requires a Map-Reduce cycle on compressed data and a rule generation phase. According to the data format, all nodes can complete their processes independently. In addition, Kavosh uses an in-memory database in the Reducer and rule generation nodes, which causes a reduction in $\mathrm{I} / \mathrm{O}$ time. According to the above description, Table 16 shows the execution time for each method.

Figure 9 shows the total execution time for each method. 
Table 17 Memory usage

\begin{tabular}{ll}
\hline Method name & $\begin{array}{l}\text { Used table } \\
\text { data volume } \\
\text { (TB) }\end{array}$ \\
\hline FiDoop & 39.9 \\
Sequence-Growth & 70 \\
Kavosh & 70 \\
\hline
\end{tabular}

Table 18 Hadoop configuration

\begin{tabular}{lc}
\hline Method & $\begin{array}{c}\text { Balance } \\
\text { Factor } \\
\text { (s) }\end{array}$ \\
\hline FiDoop & 800 \\
Sequence-Growth & 3230 \\
Kavosh & 15 \\
\hline
\end{tabular}

\section{Compression}

In this part of the evaluation, the Kavosh data volume is compared with those of FiDoop and Sequence-Growth. In FiDoop and Sequence-Growth, no compression is performed on the input data, whereas the data are compressed in Kavosh due to the conversion of the input data to the Kavosh data format. The compression rates are different: if fields have fewer discrete values, the compression rate reaches ninety percent, but Kavosh compresses data by an average of approximately $57 \%$. The data compression in the Kavosh data format is due to the conversion of string and number values to the bit data type. In the Kavosh method, no data replication or collocation is required for performance issues. In addition, data compression helps Kavosh retrieve more data in the data retrieval phase. Table 17 shows the memory usage for different methods.

\section{Load balancing}

In this section, the load balancing of various methods is investigated. To calculate the load balance, the length of time during which a processor has a CPU usage of over eighty percent while other CPUs have CPU usages of under thirty percent is calculated. In this paper, this measure is called Balance_Factor. Using this definition, the results in Table 18 were obtained.

\section{Real traffic data of a mobile operator}

In this section, the proposed method is evaluated using real traffic data of a mobile value-added service (VAS) provider. The evaluation data include more than $60,000,000$ subscribers, together with their services. The subscribers used 3000 services and all the subscribers' activities were considered in the evaluation. The mobile operator needs accurate information about their customers' favourites to be able to provide appropriate VAS suggestions. Due to the high volume of information, existing association rule algorithms would take a long time to execute and, therefore, are not suitable. In this evaluation, high-dimensional data items were used. If all the combinations of rules were applied, $2^{3000}-1$ mapping fields would have to be added to each rule that is generated 
Table 19 Input data format

\begin{tabular}{ll}
\hline MobileNumber & ServicelD \\
\hline 09121450111 & 1041 \\
09121450111 & 58 \\
09121450111 & 971 \\
09121450111 & 119 \\
09123895004 & 971 \\
09191005069 & 113 \\
$\ldots$ & $\ldots$ \\
\hline
\end{tabular}

in the rule generation layer. Such a large number of rules is neither necessary nor calculable. Based on additional information obtained about the services, a maximum of ten services is required for recommending VAS. Thus, rules with a maximum length of ten must be generated.

The input data format is as shown in Table 19. The first column is the subscriber mobile number and the second column is the service's ID.

We converted Table 18 to a binominal format. Finally, a $<$ Key,Value $>$ pair is created. The Key is the mobile number, and the value is a bit stream with a length of 2999 (Number of VAS services - 1 (Target Field)). Kavosh was deployed on the hardware nodes with the specifications detailed in "TPC-DS" section.

In the first step, the customer service information table is converted to the Kavosh format. In this phase, one service is considered as a target field. The relationships between this service and other services are extracted as rules. The customer service information table is distributed over 70 nodes. The conversion time is 1200 s. Fifty servers are used for the Mapper, one server is used for the Reducer and sixty servers are used for the rule generation layer.

Table 20 presents the parameters that are used for the association rule algorithm.

Table 21 shows execution details for the three methods.

Table 20 Association rule parameters

\begin{tabular}{ll}
\hline Parameter name & $\begin{array}{l}\text { Parameter } \\
\text { value (\%) }\end{array}$ \\
\hline Confidence & 85 \\
Support & 0.1 \\
\hline
\end{tabular}

Table 21 Execution details

\begin{tabular}{lccc}
\hline Method name & Map (s) & Reduce (s) & $\begin{array}{l}\text { Rule } \\
\text { generation } \\
\text { (s) }\end{array}$ \\
\hline FiDoop & 206 & 199 & 0 \\
Sequence-Growth & 779 & 750 & 0 \\
Kavosh & 75 & 71 & 119 \\
\hline
\end{tabular}




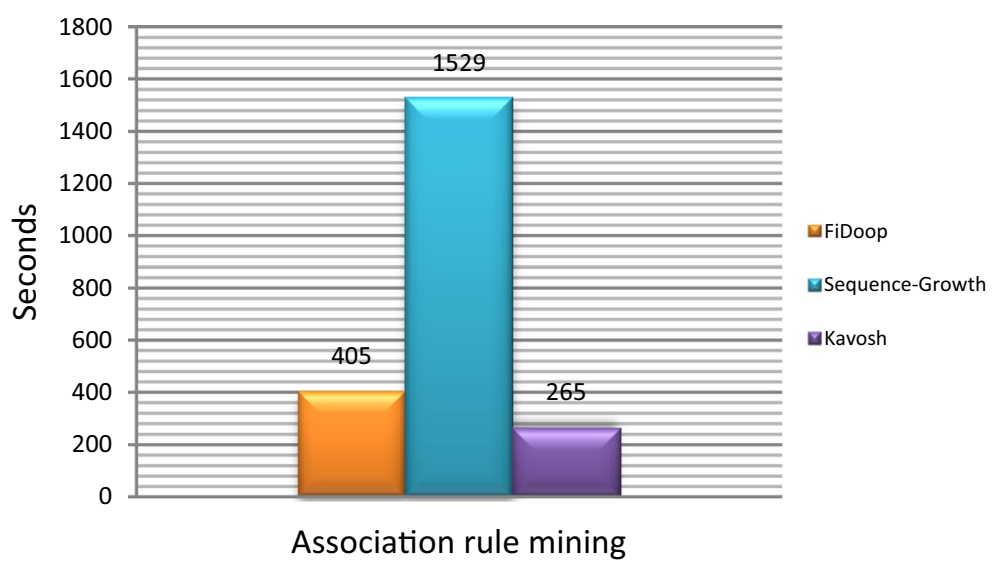

Fig. 10 Association rule mining execution time

Table 22 Memory usage

Method name

\begin{tabular}{ll} 
& $\begin{array}{l}\text { volume used } \\
\text { (TB) }\end{array}$ \\
\hline FiDoop & 19 \\
Sequence-Growth & 40 \\
Kavosh & 40 \\
\hline
\end{tabular}

\section{Table 23 Load balancing}

\begin{tabular}{lc}
\hline Method & $\begin{array}{c}\text { Balance } \\
\text { Factor } \\
\text { (s) }\end{array}$ \\
\hline FiDoop & 683 \\
Sequence-Growth & 2800 \\
Kavosh & 17 \\
\hline
\end{tabular}

Figure 10 shows the total execution time for each method.

\section{Compression}

Table 22 shows the memory usage for the three methods.

Load balancing

Table 23 shows the values of Balance_Factor for the three methods 


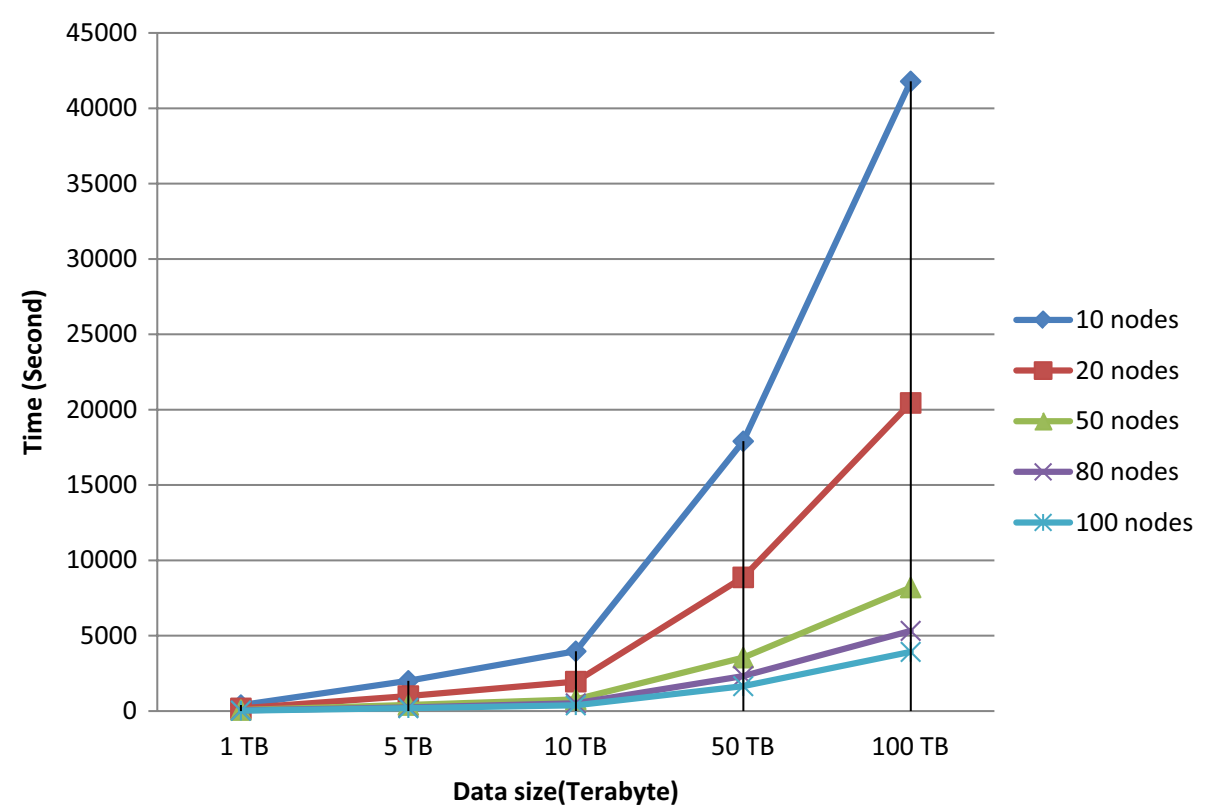

Fig. 11 Kavosh scalability

\section{Scalability}

In this section Kavosh scalability is evaluated. Different data sizes(TPC-DS) and various number of nodes are investigated and the results are depicted as Fig. 11. The results show that by adding more nodes and scale out data over nodes less time is required for data processing.

\section{Conclusion}

In this paper, the Kavosh method is proposed. Kavosh is a Map-Reduce-based association rule mining method that can manage an immense amount of data. This method converts input data into the Kavosh format, which is a unified and compressed format. Using the Kavosh data format, data can be distributed over nodes, and nodes do not require additional data from other nodes. Kavosh also compresses input data and facilitates data management. The main advantage of the proposed method is the omission of iterations for extracting rules. This is a substantial achievement because writing intermediate results to a disk and retrieving them for the next iteration is the most time-consuming portion of association rule mining. Another important achievement of the proposed method is the balanced process for each node. Because of the unified data format, each node is allocated the same number of rows in the homogenous nodes; thus, the process is equally divided among the nodes. By modifying Hadoop, the proposed method was implemented. Kavosh was compared with prominent association rule mining methods and achieved a faster execution time.

Kavosh is not suitable for high-dimensional data because creating all the input field combinations requires many nodes in the data generation layer. However, Kavosh is completely usable for normal data (not high-dimensional data) and can generate rules of a predefined length if other rule lengths are not required. 
Data format unification can be applied to problems in other fields. This method can be used in data warehouse like Aras and Atrak methods [22, 23], graph processing [24], integrating multidimensional data sources [25] and specific problems like finding patient similarity [26]. For future works, this method can also be used for interactive query processing, online data mining and stream processing.

\section{Authors' contributions}

All authors contributed equally. All authors read and approved the final manuscript

\section{Acknowledgements}

None.

\section{Ethics approval and consent to participate and Consent for publication}

In submitting an article to any of the journals published by SpringerOpen I certify that: (1) I am authorized by my coauthors to enter into these arrangements. (2) I warrant, on behalf of myself and my co-authors, that: (i) the article is original, has not been formally published in any other peer-reviewed journal, is not under consideration by any other journal and does not infringe any existing copyright or any other third party rights; (ii) I am/we are the sole author(s) of the article and have full authority to enter into this agreement and in granting rights to Springer are not in breach of any other obligation; (iii) the article contains nothing that is unlawful, libellous, or which would, if published, constitute a breach of contract or of confidence or of commitment given to secrecy; (iv)l/we have taken due care to ensure the integrity of the article. To my/our - and currently accepted scientific — knowledge all statements contained in it purporting to be facts are true and any formula or instruction contained in the article will not, if followed accurately, cause any injury, illness or damage to the user. (3) I, and all co-authors, agree that the article, if editorially accepted for publication, shall be licensed under the Creative Commons Attribution License 4.0. If the law requires that the article be published in the public domain, I/we will notify Springer at the time of submission, and in such cases the article shall be released under the Creative Commons 1.0 Public Domain Dedication waiver. For the avoidance of doubt it is stated that "Introduction" and "Related works" sections of this license agreement shall apply and prevail regardless of whether the article is published under Creative Commons Attribution License 4.0 or the Creative Commons 1.0 Public Domain Dedication waiver. (4) I, and all co-authors, agree that, if the article is editorially accepted for publication in Chemistry Central Journal, Chemical and Biological Technologies in Agriculture, Geochemical Transactions, Heritage Science, Journal of Cheminformatics, or Sustainable Chemical Processes, data included in the article shall be made available under the Creative Commons 1.0 Public Domain Dedication waiver, unless otherwise stated. For the avoidance of doubt it is stated that "Introduction", "Related works" and "Methods" sections of this license agreement shall apply and prevail.

\section{Competing interests}

The authors declare that they have no competing interests.

\section{Availability of data and materials}

http://www.tpc.org/TPC_Documents_Current_Versions/download_programs/tools-download-request.asp?bm_ type $=$ TPC-DS\&bm_vers $=2.7 .0 \&$ mode $=$ CURRENT-ONLY.

\section{Funding}

None.

\section{Publisher's Note}

Springer Nature remains neutral with regard to jurisdictional claims in published maps and institutional affiliations.

Received: 22 March 2018 Accepted: 18 June 2018

Published online: 14 July 2018

\section{References}

1. Dean J, Ghemawat S. MapReduce: simplified data processing on large clusters. Commun ACM. 2008;51(1):107-13.

2. Zaki MJ. Parallel and distributed association mining: a survey. IEEE Concurr. 1999;7(4):14-25.

3. Pramudiono I, Kitsuregawa M. FP-tax: tree structure based generalized association rule mining. In: Proceedings of the 9th ACM SIGMOD workshop on research issues in data mining and knowledge discovery. New York: ACM; 2004. p. 60-3.

4. Oruganti S, Ding Q, Tabrizi N. Exploring Hadoop as a platform for distributed association rule mining. In: Future computing 2013 the fifth international conference on future computational technologies and applications; 2013. p. 62-7.

5. Kovacs F, Illés J. Frequent itemset mining on hadoop. In: 2013 IEEE 9th international conference on computational cybernetics (ICCC). New York: IEEE; 2013. p. 241-5.

6. Li N, Zeng L, He Q, Shi Z. Parallel implementation of apriori algorithm based on mapreduce. In: 2012 13th ACIS international conference on software engineering, artificial intelligence, networking and parallel and distributed computing (SNPD). New York: IEEE; 2012. p. 236-41.

7. Yang XY, Liu Z, Fu Y. MapReduce as a programming model for association rules algorithm on Hadoop. In: 2010 3rd international conference on information sciences and interaction sciences (ICIS). New York: IEEE; 2010. p. 99-102. 
8. Li L, Zhang M. The strategy of mining association rule based on cloud computing. In: 2011 international conference on business computing and global informatization (BCGIN). New York: IEEE; 2011. p. 475-8.

9. Lin MY, Lee PY, Hsueh SC. Apriori-based frequent itemset mining algorithms on MapReduce. In: Proceedings of the 6th international conference on ubiquitous information management and communication. New York: ACM; 2012. p. 76.

10. Xun Y, Zhang J, Qin X. Fidoop: parallel mining of frequent itemsets using mapreduce. IEEE Trans Syst Man Cybern Syst. 2016:46(3):313-25.

11. Barkhordari M, Niamanesh M. ScadiBino: an effective MapReduce-based association rule mining method. In: Proceedings of the sixteenth international conference on electronic commerce. New York: ACM; 2014. p. 1.

12. Yu KM, Zhou J, Hong TP, Zhou JL. A load-balanced distributed parallel mining algorithm. Expert Syst Appl. 2010:37(3):2459-64

13. Li H, Wang Y, Zhang D, Zhang M, Chang EY. Pfp: parallel fp-growth for query recommendation. In: Proceedings of the 2008 ACM conference on recommender systems. New York: ACM; 2008. p. 107-14.

14. Bechini A, Marcelloni F, Segatori A. A MapReduce solution for associative classification of big data. Inf Sci. 2016:332:33-55.

15. Yang L, Shi Z, Xu LD, Liang F, Kirsh I. DH-TRIE frequent pattern mining on Hadoop using JPA. In: 2011 IEEE international conference on granular computing (GrC). New York: IEEE; 2011. p. 875-8.

16. Tlili R, Slimani Y. A novel data partitioning approach for association rule mining on grids. Int J Grid Distributed Comput. 2012;5(4):1-20.

17. Riondato M, DeBrabant JA, Fonseca R, Upfal E. PARMA: a parallel randomized algorithm for approximate association rules mining in MapReduce. In: Proceedings of the 21 st ACM international conference on Information and knowledge management. New York: ACM; 2012. p. 85-94

18. Yu KM, Zhou J. Parallel TID-based frequent pattern mining algorithm on a PC Cluster and grid computing system. Expert Syst Appl. 2010;37(3):2486-94.

19. Moens S, Aksehirli E, Goethals B. Frequent itemset mining for big data. In: 2013 IEEE international conference on Big Data. New York: IEEE; 2013. p. 111-8.

20. Liang YH, Wu SY. Sequence-growth: A scalable and effective frequent itemset mining algorithm for big data based on MapReduce framework. In: 2015 IEEE international congress on Big Data (BigData Congress). New York: IEEE; 2015. p. 393-400.

21. Chaudhary S, Sharma A, Singh R, Kumar P. Lexicographic logical multi-hashing for frequent itemset mining. In: 2015 international conference on computing, communication and automation (ICCCA). New York: IEEE; 2015. p. 563-8.

22. Barkhordari M, Niamanesh M. Atrak: a MapReduce-based data warehouse for big data. J Supercomput. 2017;73:4596-610

23. Barkhordari M, Niamanesh M. Aras: a method with uniform distributed dataset to solve data warehouse problems for big data. Int J Distributed Syst Technol (IJDST). 2017;8(2):47-60.

24. Barkhordari M, Niamanesh M. ScaDiGraph: a MapReduce-based method for solving graph problems. J Inform Sci Eng. 2017;33(1):143-58.

25. Barkhordari M, Niamanesh M. Arvand: a method to integrate multidimensional data sources into big data analytic structures. J Inf Sci Eng. 2018;34(2):505-18.

26. Barkhordari M, Niamanesh M. ScaDiPaSi: an effective scalable and distributable MapReduce-based method to find patient similarity on huge healthcare networks. Big Data Res. 2015;2(1):19-27.

\section{Submit your manuscript to a SpringerOpen ${ }^{\circ}$ journal and benefit from:}

- Convenient online submission

- Rigorous peer review

- Open access: articles freely available online

- High visibility within the field

Retaining the copyright to your article

Submit your next manuscript at $\mathbf{s p r i n g e r o p e n . c o m ~}$ 